\title{
Urinary tract infection after radical cystectomy: a vexing problem despite prophylactic antibiotics
}

\author{
Joseph Y. Clark, Jay D. Raman \\ Department of Surgery (Urology), Penn State Health, Hershey, PA, USA \\ Correspondence to: Joseph Y. Clark. Department of Surgery (Urology), Penn State Health, Hershey, PA 17033, USA. \\ Email: jclark13@pennstatehealth.psu.edu. \\ Provenance: This is an invited article commissioned by Section Editor Xiao Li (Department of Urology, Jiangsu Cancer Hospital \& Jiangsu Institute \\ of Cancer Research \& Nanjing Medical University Affiliated Cancer Hospital, Nanjing, China). \\ Comment on: Haider M, Ladurner C, Mayr R, et al. Use and duration of antibiotic prophylaxis and the rate of urinary tract infection after radical \\ cystectomy for bladder cancer: Results of a multicentric series. Urol Oncol 2019;37:300.e9-300.e15.
}

Submitted Jun 30, 2019. Accepted for publication Jul 09, 2019.

doi: $10.21037 /$ tau.2019.07.07

View this article at: http://dx.doi.org/10.21037/tau.2019.07.07

Radical cystectomy (RC) with pelvic lymph node dissection, preceded by cisplatin-based neoadjuvant chemotherapy in appropriate patients, remains the standard treatment of muscle invasive bladder cancer (1). Despite improvement in perioperative care pathways with an associated trend towards lower mortality, RC remains a complex and often morbid operation with significant risk to patients (2). Early infectious complication rates after RC and urinary diversion based on several large contemporary institutional cohorts ranges from $7-30 \%$ and is a significant concern postprocedure (3).

The article by Haider et al. (4) published in Urologic Oncology: Seminars and Original Investigations assessed the rate of urinary tract infection (UTI) in a cohort of patients who had undergone open RC between 2009 and 2015. The authors performed a retrospective review of the charts of 217 patients who had open RC at one institution and classified the severity of the UTI based on European Association of Urology (EAU) Section of Infections in Urology classification system (5). As expected for a single institution retrospective study, there was fair consistency in use of prophylactic antibiotics, with most patients receiving a combination of metronidazole and a cephalosporin. Interestingly, the median duration of prophylactic antibiotics was 7 days and after cessation of the prophylactic antibiotics, over half $(51.6 \%)$ of patients were prescribed additional antibiotics. Of the patients, $42(19 \%)$ had a postoperative UTI diagnosed within 30 days of surgery, but more significantly half of these patients had urosepsis or uroseptic shock. When the bacterial species were identified, Enterococcus was the most common (25.7\%), followed by Klebsiella (15.8\%), Candida (14.9\%), and E. coli (13.9\%). After multivariable regression analysis of the typical risk factors and clinicopathologic variables associated with infection, only those who had a continent urinary diversion had a higher risk of developing a UTI.

This paper brings to light the question of what antibiotics should be used for prophylaxis, and how long should the antibiotics be given in patients undergoing RC. Unlike most other urologic procedures, in RC, both the urinary and gastrointestinal tracts require manipulation as well as entry into their respective cavities and therefore both the American Urological Association (AUA) (6) and EAU (7) accordingly recommend antibiotic perioperative prophylaxis for this procedure; however, there is great variation in choice and duration of antibiotics. In a retrospective series of 52,349 patients who underwent RC in the United States, only $15 \%$ of the patients received the recommended antibiotics based on the guidelines (8). In that study, the most common non soft tissue infectious event was a UTI in $12 \%$ of patients followed by sepsis in $8.2 \%$. Furthermore, these authors found that an extended duration of antibiotics had no effect on outcomes and that regimens with penicillin-based agents combined with 
$\beta$-lactamase inhibitors were associated with lower infectious rates. In general, the present study (4) did follow the AUA/ EAU guidelines with regards to prophylactic antibiotic selection, although the length of prophylaxis in more than half of the cases went beyond $24 \mathrm{~h}$ and additional antibiotics were prescribed in $51.6 \%$ of patients after the cessation of the perioperative course of antibiotics.

In an effort to decrease the rates of UTIs following $\mathrm{RC}$, other groups have administered a prolonged course of antibiotics. Specifically, Werntz et al. (9) prescribed antibiotic prophylaxis for all patients discharged to home after RC; their protocol was to obtain a urine culture prior to discharge and prescribe either trimethoprimsulfamethoxazole (TMP-SMX; $160 \mathrm{mg} / 800 \mathrm{mg}$ ), nitrofurantoin $100 \mathrm{mg}$, or ciprofloxacin $250 \mathrm{mg}$ daily depending on individual contraindication and tolerability. Of note, if the urine culture did grow bacteria, they were discharged on 7 days of culture specific antibiotics before resuming their prophylaxis. They standardized their ureteral stent removal at 3 weeks following the RC. Their results were impressive: only $12 \%$ of the patients in the prophylactic antibiotic group had a UTI compared to $36 \%$ in those receiving no antibiotics. Urosepsis was significantly less common in the prophylactic antibiotic group (2\% vs. $17 \%)$. Speciation of organisms responsible for the UTIs in this study were Enterococcus (32\%), E coli (27\%), Pseudomonas (14\%), and Candida albicans (9\%).

Another consideration in use of antibiotics concerns Clostridium difficile infection (CDI). Single institution series of CDI in patients undergoing $\mathrm{RC}$ averaged $5 \%$ and it is likely that there is underreporting in national datasets (10). Indeed, the antibiotic prophylaxis regimen may affect CDI rate. Liu et al. (11) showed that almost $9 \%$ of patients undergoing RC developed CDI and that use of chronic antacid therapy and long duration of antimicrobial exposure were independent predictors. The present study (4) did not report any case of CDI and the study by Werntz et al. (9) interestingly, reported only one case of CDI in the group that did not have a prolonged course of antibiotics.

It appears that following antibiotic prophylaxis guidelines does not increase the rate of UTI; Cai et al. (12) demonstrated in a single institution study that implementing a protocol adhering to EAU guidelines for antibiotic prophylaxis for all urologic surgeries over a 2 year period, did not increase risk of infections or development of resistant organisms compared to historical controls. Overall, their subset of patients undergoing RC was low (49 before and 54 after instituting the protocol) and they found a "statistically significant reduction in the rate of antibiotic-related adverse events, with eight cases of CDI before and one case after protocol implementation" and these events mainly "occurred following major open urologic surgery (radical cystectomy)." Another finding of this study was that the length of stay for patients undergoing RC was significantly reduced from 18 to 13 days although the authors admit this could also be due to "improved postoperative management as a result of protocol implementation." Thus, it seems possible through antibiotic stewardship, to reduce overall antibiotic use and consequent cost, and decrease the risk of development of resistant bacterial pathogens without increasing the risk of postoperative infection.

The present study (4) concludes that continent urinary diversion had a higher risk of developing a UTI (odds ratio 5.0; $\mathrm{P}<0.001)$ compared with those who had an incontinent urinary diversion. This is in keeping with other studies such as by Mano et al. (13) who found that during the first 3 months after RC, 29\% of the patients with a neobladder and $8 \%$ of the patients with ileal conduit had a UTI episode.

One notable strength of this paper is the profile of bacteria causing the UTI specifically with Enterococcus being the most common organism and would not be covered by the standard prophylaxis. Enterococcus was also the most common bacteria causing UTIs in the study by Werntz et al. (9). A study from the Mayo Clinic by Parker et al. (14) on patients treated with RC over a 10 -year period showed that patients received a variety of perioperative antibiotic regimens and about $38 \%$ of the UTIs were polymicrobial, and of organisms cultured, the most common were gram positive (Enterococcus and Staphylococcus aureus making up the majority). The most common gram-negative organisms were E. coli and Pseudomonas aeruginosa, and surprisingly, $24 \%$ of the UTIs in their population were fungal.

This paper is retrospective in nature and has a relatively small sample size which is a problem with other papers describing UTIs after RC. This, combined with the expected bacteriuria and the lack of a standardized definition of UTI in patients with a urinary diversion, the difficulty in confirming the diagnosis of a UTI in this subset of patients, various perioperative antibiotic choices and differing lengths of treatment are confounding factors and make general recommendations difficult. It also calls to light the need for larger, prospective studies with standardized definitions and protocols to include choice of prophylactic antibiotics and timing of ureteral stent or Foley removal. 
Now with the recognition that enhanced recovery after surgery (ERAS) regimens in patients undergoing RC may improve outcomes, and are being adopted by more centers world-wide (15), there may yet be an opportunity for high volume centers to collaborate and adopt an ERAS regimen which includes antibiotic prophylaxis and minimizes the variance and prospectively study this vexing problem.

So what strategy should one use to guide antibiotic prophylaxis and treatment for patients undergoing $\mathrm{RC}$ given these disparate studies? Guidelines based prophylaxis regimens with some tailoring based on an institution's local antibiogram is a logical method to select the type and duration of prophylaxis prior to RC. Perhaps routinely obtaining urine cultures in postoperative patients who are asymptomatic, especially in those with continent urinary diversions or those with foreign bodies such as Foley catheters, ureteral stents, or nephrostomy tubes would allow more accurate and earlier treatment if the patient later became symptomatic. It seems that patients who have continent diversions indeed have a higher risk of UTI so one should be more vigilant in these patients after RC. If patients develop a UTI in the postoperative period, broad spectrum antibiotics which would cover gram positive organisms and perhaps fungi are appropriate while awaiting final cultures. Not directly related to antibiotic selection, but optimizing the patient's nutritional status and glycemic control and smoking cessation preoperatively, and excellence and efficiency in operative technique are all factors that may decrease UTI rates as well as other complications.

\section{Acknowledgments}

None.

\section{Footnote}

Conflicts of Interest: The authors have no conflicts of interest to declare.

Ethical Statement: The authors are accountable for all aspects of the work in ensuring that questions related to the accuracy or integrity of any part of the work are appropriately investigated and resolved.

\section{References}

1. Chang SS, Bochner BH, Chou R, et al. Treatment of Non-
Metastatic Muscle-Invasive Bladder Cancer: AUA/ASCO/ ASTRO/SUO Guideline. J Urol 2017;198:552-9.

2. Hounsome LS, Verne J, McGrath JS, et al. Trends in operative caseload and mortality rates after radical cystectomy for bladder cancer in England for 1998-2010. Eur Urol 2015;67:1056-62.

3. Anderson CB, McKiernan JM. Surgical Complications of Urinary Diversion. Urol Clin North Am 2018;45:79-90.

4. Haider M, Ladurner C, Mayr R, et al. Use and duration of antibiotic prophylaxis and the rate of urinary tract infection after radical cystectomy for bladder cancer: Results of a multicentric series. Urol Oncol 2019;37:300. e9-300.e15.

5. Johansen TE, Botto H, Cek M, et al. Critical review of current definitions of urinary tract infections and proposal of an EAU/ESIU classification system. Int J Antimicrob Agents 2011;38 Suppl:64-70.

6. Wolf JS, Bennett CJ, Dmochowski RR, et al. Best Practice Policy Statement on Urologic Surgery Antimicrobial Prophylaxis. J Urol 2008;179:1379-90.

7. Grabe M, Bartoletti R, Bjerklund-Johansen TE, et al. Guidelines on Urological Infections. European Association of Urology 2015. Available online: https:// uroweb.org/wp-content/uploads/19-Urologicalinfections_LR2.pdf

8. Krasnow RE, Mossanen M, Koo S, et al. Prophylactic Antibiotics and Postoperative Complications of Radical Cystectomy: A Population Based Analysis in the United States. J Urol 2017;198:297-304.

9. Werntz RP, Martinez-Acevedo A, Amadi H, et al. Prophylactic antibiotics following radical cystectomy reduces urinary tract infections and readmission for sepsis from a urinary source. Urol Oncol 2018;36:238.e1-238.e5.

10. Miller R, Heinlen JE. Reported rates of clostridium difficile following radical cystectomy in national datasets compared to individual institutions. Urol Oncol 2018;36:526.e7-526.e11.

11. Liu NW, Shatagopam K, Monn MF, et al. Risk for Clostridium difficile infection after radical cystectomy for bladder cancer: Analysis of a contemporary series. Urol Oncol 2015;33:503.e17-22.

12. Cai T, Verze P, Brugnolli A, et al. Adherence to European Association of Urology Guidelines on Prophylactic Antibiotics: An Important Step in Antimicrobial Stewardship. Eur Urol 2016;69:276-83.

13. Mano R, Baniel J, Goldberg H, et al. Urinary tract infections in patients with orthotopic neobladder. Urol 
Oncol 2014;32:50.e9-14.

14. Parker WP, Toussi A, Tollefson MK, et al. Risk Factors and Microbial Distribution of Urinary Tract Infections Following Radical Cystectomy. Urology 2016;94:96-101.
15. Pang KH, Groves R, Venugopal S, et al. Prospective Implementation of Enhanced Recovery After Surgery Protocols to Radical Cystectomy. Eur Urol 2018;74:e66.

Cite this article as: Clark JY, Raman JD. Urinary tract infection after radical cystectomy: a vexing problem despite prophylactic antibiotics. Transl Androl Urol 2019;8(Suppl 5):S510-S513. doi: 10.21037/tau.2019.07.07 